\title{
Optimizing Feed Withdrawal Time to Assess Broiler Stress and Welfare
}

\author{
Hamidu, J. A*., Agbehadzi, R. K., Amexo, V. E., Brown, C. A, Adomako, K. \\ Department of Animal Science, Kwame Nkrumah University of Science and Technology, \\ Kumasi, Ghana \\ *jahamidu@gmail.com
}

\begin{abstract}
The study investigates effects of feed withdrawal time on stress indices in broilers. Ross 308 broilers $(n=10)$, aged 6 weeks were obtained from a commercial farm and transported to KNUST-Kumasi. The birds were placed in pens and provided water ad-libitum but without feed. Sample of birds $(n=2)$ were withdrawn randomly on arrival and every 4 hours following feed withdrawal (0 hours, 4 hours, 8 hours, 12 hours and 16 hours) corresponding to 4pm, 8pm, 12am, 4am and 8am. Blood samples were drawn from the brachial veins under the wings of the two birds using a syringe and pooled together into an evacuated heparinized tubes with EDTA and Serum. The EDTA tubes were shaken to prevent coagulation of the blood. The blood samples were stored at $4^{\circ} \mathrm{C}$ and analysed at the Clinical analysis laboratory, KNUST for the concentrations of hormones: triiodothyronine (T3), thyroxine (T4), thyroid stimulating hormones (TSH) as indicators of stress. The T3/T4 ratios were calculated. Relationships between blood thyroid hormone levels and feed withdrawal time impact on body weight were established. The results showed a decrease in T3 and T4 concentration during the first four hours. After the 4 hours T3 rapidly increased and after 8 hours feed of withdrawal it decreased sharply. The T4 gradual increased may indicate increased stress and perhaps preparation for conversion of T4 to T3, the active form of the hormone to increased metabolism to compensate for increasing feed withdrawal loss. The T3/T4 ratio and TSH of 1.71 and 0.1 was highest and lowest respectively at 8 hours. There was high positive relationship between feed withdrawal time and T4 concentration $\left(Y=0.5275 x+5.94, R^{2}=0.7431\right)$. Body weight decreased with increasing T4 concentration $\left(y=-0.0469 x+2.2535 R^{2}=0.45\right)$. As T4 increases following feed withdrawal, body weight reduces by $4.6 \%$. The study revealed that feed withdrawal duration of 16 hours leads to $44.9 \%$ decrease in body weight of broilers. It appears that beyond 8 hours of feed withdrawal birds were physiologically stressed and could be subject to negative welfare conditions which leads to body weight loss and reduced meat yield.
\end{abstract}

Keywords: Broilers, feed withdrawal, physiological stress, welfare and meat yield.

\section{INTRODUCTION}

In an effort to reduce carcass contamination at processing plants, commercial producers have, for many years, typically pulled their birds off feed prior to catching, loading, and transporting the broilers to the processing plant [1]. Producers choice to remove feed are based on research that shows withdrawing feed prior to transport and processing reduces the amount of ingesta in the gastrointestinal tract and reduces the incidence of torn or ruptured gastrointestinal tracts, and decreasing the likelihood of carcass contamination [2]. The timeline for feed and sometimes water withdrawal can be anywhere from 2 to 24 hours. The ideal withdrawal period, however, should be short enough to avoid considerable losses in live weights or carcass yields, but long enough to allow the digestive tract to become empty [3].

However, feed withdrawal affects a lot of metabolic processes. Feed deprivation causes a shift from anabolism to catabolism, from lipogenesis to lipolysis, and a reduced metabolic rate [4]. Consequently, feed withdrawal can cause bodyweight loss between 0.22 and $0.56 \%$ per hour [5]. Similar reports show significant increase in live broiler weight losses from 1.3 to $5.3 \%$ in 18 hourperiod [6]. Additionally, the drops in dressed and chilled carcass yields were higher, with dressed carcass yields of $65.2 \%$ after 3 hours and $63.8 \%$ after 12 hours following feed withdrawal. It is also evident that, feed withdrawal induces behavioral and physiological responses, indicating that broilers probably suffer from stress andbreaking a lot of welfare rules of the birds [7], [8] and [9].

A variety of stressors used to study stress responses in poultry species include mediation of the adrenal glands directly by exogenous administration of adrenocorticotropin (ACTH) and exogenous 
administration of steroid moieties, including corticosterone (CS)[10]. The two most common indicators of the stress condition in birds are elevated blood plasma CS and increased circulating Heterophil/lymphocyte $(\mathrm{H} / \mathrm{L})$ [11]. Therefore, the objective for the study was todetermine the relationship between feed withdrawal lengths in broilers and stressful indicators that may be used by birds in communicating stress physiologically and determine the impact of stress on body weight and meat yield.

\section{MATERIALS AND MeTHODS}

\subsection{Location and Duration of Experiment}

The experiment was conducted at the Poultry section of the Animal Science Department- Kwame Nkrumah University of Science and Technology (KNUST), Kumasi. The experiment lasted for 17 hours. Birds were handled humanely following the Department of Animal Science, KNUST laid down regulations for handling animals for experiments.

\subsection{Experimental Birds}

A total of 10 commercial Ross 308 broilers aged 6 weeks with an average weight of $1.8 \mathrm{~kg}$ were obtained from Jokas Farms, Abrepo-Kumasi, placed in plastic cages and transported to the experimental site for about 30 minutes with all windows on the vehicle opened for maximum aeration. The experimental birds were given ad libitum access to water on arrival. The birds were immediately put under feed withdrawal of 0 hour [4pm], 4 hours [8pm], 8 hours[12am], 12 hours [4am], and 16 hours [8am]. Two birds were randomly selected for every 4 hours interval and blood samples drawn from the brachial vein of the birds under the wings and placed into evacuated heparinized tubes. Blood was drawn by the use of syringes. The syringes were changed after every treatment. The blood samples per birds per treatment were placed in 2 tubes (EDTA and Serum); each tube contained blood from both birds. The EDTA tubes were shaken to prevent coagulation of the blood. The blood samples were stored at $4^{\circ} \mathrm{C}$ during the experiment to prevent coagulation.

\subsection{Blood Analysis}

The blood samples were taken to the Clinical Analysis Laboratory (CAn-Lab) of the Department of Biochemistry, KNUST for analysis. Each blood sample was analyzed for the concentration of thyroid hormones; thyroxine $\left(\mathrm{T}_{4}\right)$, Triiodothyronine $\left(\mathrm{T}_{3}\right)$, Thyroid Stimulating Hormone (TSH). Quantitative values of plasma T3, T4 and TSH concentrations were determined by the solid-phase enzyme immunoassay. This Kit is designed for the measurement of free triiodothyronine ( $\mathrm{fT}_{4}$ ), free thyroxin $\left(\mathrm{fT}_{3}\right)$ and TSH in blood serum or plasma. The triiodothyronine and thyroxin test was based on competition enzyme immunoassay principle. Tested specimen to be analyzed for T4 was placed into the microwells coated by specific murine monoclonal to T4-antibodies simultaneously with conjugated free triiodothyronine (fT4) peroxidase. The specimen for T3 was also placed in microwells coated by specific rabbit polyclonal to T3-antibodies simultaneously with conjugated fT3-peroxidase. TSH was tested based on two-sited sandwich enzyme immunoassay principle. The tested specimen was placed into the microwells coated by specific murine monoclonal $\beta$ chain TSH-antibodies. (XEMA Co., Ltd, Instruction Manual: Version 210, 2011).

\subsection{Data Analysis}

The results were analyzed using Microsoft Excel 2013 and Regression determined. The ratio of T3 and T4 was calculated.

\section{RESULTS AND DisCUSSION}

Following feed withdrawal T4 concentration increased linearly especially from 4 hours till 16 hours (Figure 1). Similar observation between feed withdrawal time and body weight has been reported previously [9]. However, the T3 was low at the beginning of the feed withdrawal experiment, then peak at 8 hours and then declined (Figure 1). Since higher level of T3 is an indication of increased metabolism (Sarne, 2001) [12], the bird's metabolism followed a normal process which increased until 8 hours but beyond that T3 decreased as a result of the stress of hunger and so metabolism dropped. This could be explained by the increasing T4 in the blood as feed withdrawal time increased, a response which indicates that stress is settling into the body.

Giving a detailed account of the dynamics of hormonal changes during starvation and fasting, It is reported that multiple alterations in thyroid hormone regulation and metabolism occur following during caloric restriction [12]. In this case the most dramatic effect is a decrease in the serum T3 within 24-48 hours of the initiation of fasting. Because changes in the free T3 fraction are usually 
small, the absolute concentration of free T3 is also reduced, clearly into the hypothyroid range. The marked reduction in serum T3 at prolonged feed withdrawal is caused by a reduction in its generation from $\mathrm{T} 4$ rather than by an acceleration in its metabolic clearance rate. The decline in T3 concentration is accompanied by a concomitant and reciprocal change in the concentration of total and free T3. The increase in the serum $\mathrm{T} 3$ concentration tends to begin later and to return to normal at the time serum T3 is being maintained at a low level with continuous calorie deprivation.

The study show that there was a fairly strong negative correlation between increasing levels of $\mathrm{T} 4$ and the body weight of the birds, $\left(\mathrm{y}=-0.0469 \mathrm{x}+2.2535, \mathrm{R}^{2}=0.45\right)$, with respect to the increasing feed withdrawal period (Figure 2). This analysis indicates that as T4 increases and at every four hours of feed withdrawal, body weight reduces by $4.6 \%$. The study revealed that feed withdrawal duration of 16 hours leads to $44.9 \%$ decrease in body weight of broilers. The relationship between the blood serum levels of T4 and T3 in the blood as feed withdrawal period increases showed that the effects of feed withdrawal duration affected the concentration of the T4 and T3.

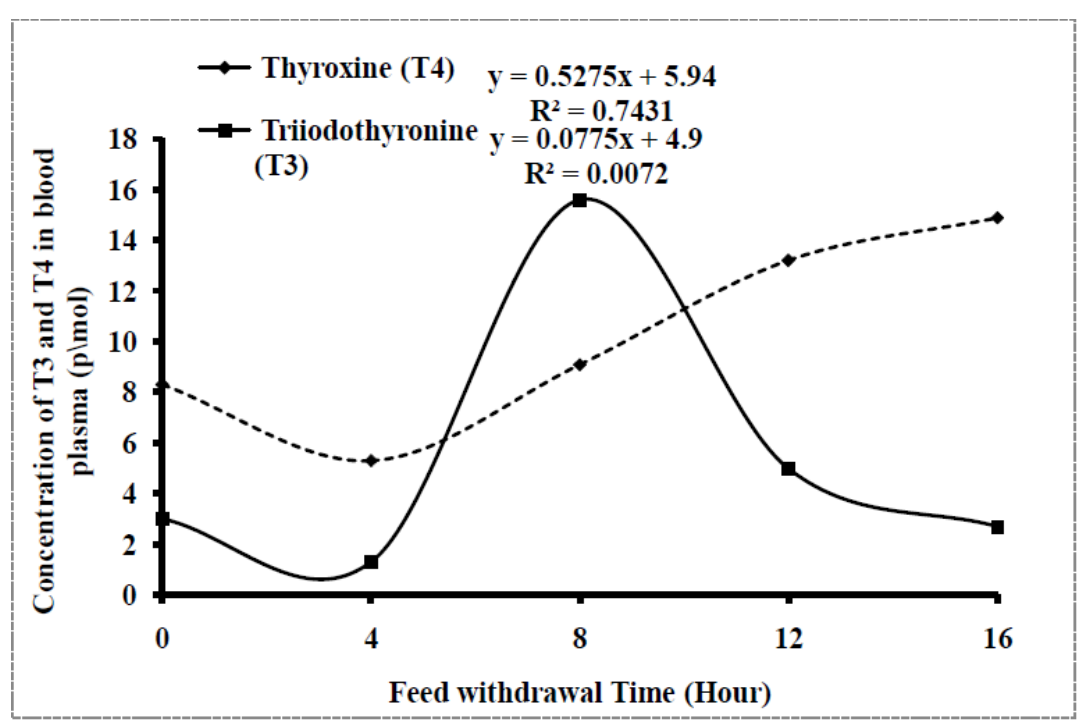

Figure 1. Concentration of triiodothyronine (T3) and thyroxine (T4) in the blood plasma of broilers following various hours of feed withdrawal prior to slaughter.

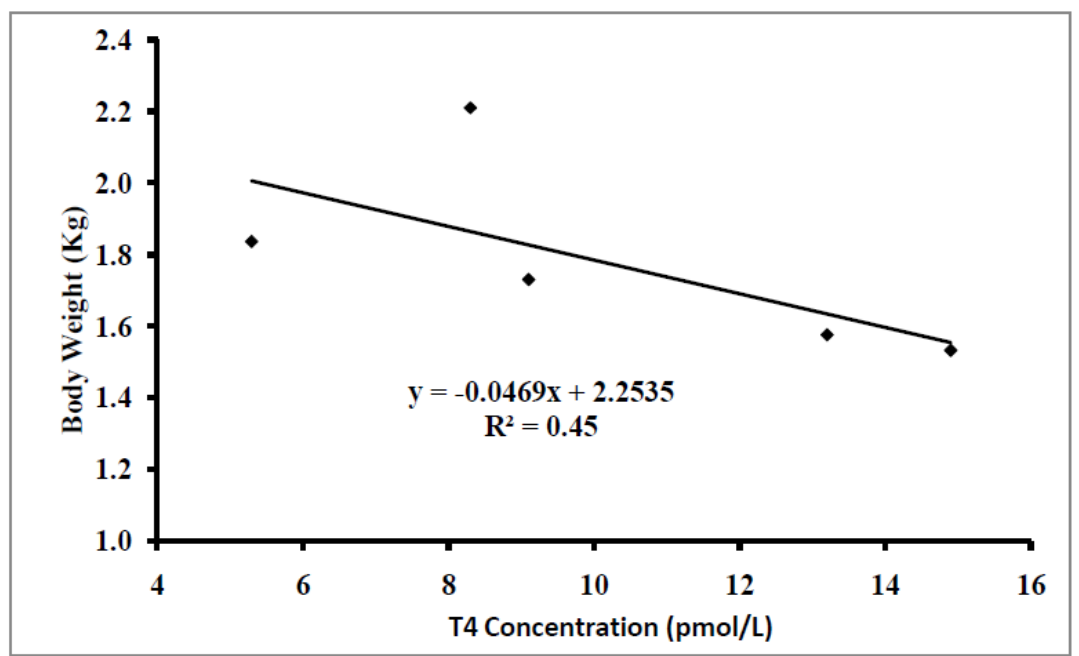

Figure 2. Weight of broilers as thyroxine (T4) concentration increases with increasing feed withdrawal time.

Table 1. Ratio of T3/T4 and the concentration of TSH $(\mu \mathrm{lU} / \mathrm{ml})$ at every feed withdrawal time.

\begin{tabular}{|l|l|l|}
\hline Feed Withdrawal Time (Hour) & T3/T4 & TSH $(\boldsymbol{\mu l U} / \mathbf{m l})$ \\
\hline 0 & 0.36 & 0.2 \\
\hline 4 & 0.25 & 0.2 \\
\hline 8 & 0.71 & 0.1 \\
\hline 12 & 0.38 & 0.3 \\
\hline 16 & 0.18 & 0.3 \\
\hline
\end{tabular}


Thyroid-stimulating hormone (TSH) is a pituitary hormone that stimulates the thyroid gland to produce thyroxine $\left(\mathrm{T}_{4}\right)$, and then triiodothyronine $\left(\mathrm{T}_{3}\right)$ which stimulates the metabolism of almost every tissue in the body. It was noted that concentration of TSH decreased to $0.1 \mu 1 \mathrm{U} / \mathrm{ml}$ at the eighth hour while T4 and T3/T4ratio was at $9.1 \mathrm{p} / \mathrm{mol}$ (Figure 1) and 0.71(Table 1) respectively. Previous studies indicate that acute stressors can cause a decrease in plasma TSH levels [13] and [14]. This could be attributed to malfunction of thyroid gland and inability to send negative feedback to ensure accurate balance of concentration of T4. TSH was highest at the twelfth and sixteenth hour with a concentration level of 0.3 . T4 at this levels were 13.2 and 14.9 respectively. Various stress factors may also elicit an increase inthyroliberin (thyrotropin-releasing hormone, TSH-RH), thyroidstimulating hormone (TSH), triiodothyronine (T3) and thyroxine (T4)[15].

In confirmation study, it has been shown that $\mathrm{T} 4$ at the fourth hour also increased simultaneously but this increase was expressed more gradually [16]. The metabolic roles of these two hormones in the bird expressed these results, because of their involvement in economizing on energy in the bird and their great importance to the growth and development of young organisms. Broilers on feed withdrawal and subjected to catching and transport intervention had lower concentration of T3 [5]. This observation confirms our result for decrease in T3 for the first four hours and last eight hours, because our broilers were also immediately put on feed withdrawal after catch and transportation to the experimental site. Broilers possibly had a decrease in T3 at the eighth hour because they started experiencing a negative TSH, T3, T4 and energy balance. Feed withdrawal affects several metabolic processes and may cause a shift from anabolism to catabolism, from lipogenesis to lipolysis and reduced metabolic rate [17].

For birds that are sold on live weight basis there will be a significant economic loss due to long feed withdrawal period. Long feed withdrawal had a negative effect on live body weight [18]. It was seen in this research that broilers placed on 14 hours feed withdrawal showed $15.2 \%$ less body weight than the control (0 hour) at 37 days. Additionally, a progressively live weight loss is was observed when feed withdrawal time increased beyond 16 hours [19].

\section{Conclusion}

The study revealed that feed withdrawal duration of 16 hours leads to $44.9 \%$ decrease in body weight as T4 concentration increases. This increases the stress level of birds leading to loss of weight and loss of meat yield and profitability.

\section{REFERENCE}

[1] Wabeck C. J., Feed and water withdrawal time relationship to processing yield and potential fecal contamination of broilers, Poult. Sci. (1972) 51(4): 1119-1121.

[2] Bilgili S. F. and Hess J. B., Tensile strength of broiler intestines as influenced by age and feed withdrawal, J. Appl. Poult. Res. (1997) 6 (3): 279-283.

[3] Veerkamp C.H., Fasting and yields of broilers, Poult. Sci. (1986) 65 (7): 1299-1304.

[4] Nijdam E., Delezie E., Lambooij E., Nabuurs M. J. A., Decuypere E. and Stegeman J. A., Feed withdrawal of broilers before transport changes plasma hormone and metabolite concentrations,Poult. Sci. (2005) 84(7):1146-52.

[5] Veerkamp C. H., The influence of fasting and transport on yields of broilers, Poult. Sci. (1978) 57 (3): 634-638.

[6] Contreras-Castillo C., Pinto A. A, Souza G. L., Beraquet N. J., Aguiar A. P., Cipolli K. M. V. A. B., Mendez M. I. and Ortega E. M., Effects of feed withdrawal periods on carcass yield and breast meat quality of chickens reared using an alternative system,J Appl. Poult. Res. (2007) 16 (4): 613-622.

[7] Freeman B. M., Glucagon: a stress hormone in the domestic fowl, Res. Vet.Sci. (1980) 28:389 390.

[8] Zuidhof, M. J., R. H. McGovern, B. L. Schneider, J. J. R. Feddes, F. E Robinson, and D. R. Korver., Implications of pre-slaughter feeding cues for broiler behavior and carcass quality, $\mathrm{J}$ Appl. Poult. Sci. (2004) 13:335-341.

[9] Hamidu, J.A., Kyeiwaa, V., Bobie-Ansah, D., Brown, C. A., Atuahene, C. C. and Adomako, K., Optimizing feed withdrawal in broilers: Effect of feed withdrawal timing on broiler carcass yield in tropics. American Research Journal of Agriculture (2015)1:7 - 15. 
[10] Puvadolpirod S. and Thaxton, J. P., Model of Physiological Stress in Chickens 1. Response Parameters, Poult. Sci.(2000) 79:363 - 369.

[11] Siegel H. S., Stress, strains and resistance, Br.Poult. Sci.(1995) 36:3 - 22.

[12] Sarne D., Effects of the environment, chemicals and drugs on thyroid function. In:L.J. DeGroot, G.Hennemanneds. The Thyroid and its Diseases. Endocrine Education, Inc., Massachusetts, (2001) Available at: http://www.ncbi.nlm.nih.gov/books/NBK285560/.

[13] Marti, O., Gavalda, A.,Jolin, T. and Armario, A., Acute stress attenuates but does not abolish circadian rhythmicity of serum thryrotropin and growth hormone in the rat, Eur. J.Endocrinol.(1996) 136:703 - 708 .

[14] Kondo, K., Harbuz, M., Levy, A. and Lightman, S., Inhibition of the hypothalamic-pituitarythyroidaxis in response to lipopolysaccharide is independent of changes in circulating corticosteroids, Neuroimmunomodulation(1997) 4:188 - 194.

[15] Iqbal, A., Decuypere, E., Abd El Azim, A., Kühn, E. R., Pre- and post-hatch high temperature exposure affects the thyroid hormones and corticosterone response to acute heat stress in growing chicken (Gallus domesticus), J.Therm. Biol. (1990) 15:149 - 153.

[16] Bobek, S., Niezgoda, J., Pierzchala, K., Litynski, P. and Sechman, A., Changes in circulating iodothyronines, cortisol and endogenous thiocyanate in sheep during emotional stress caused by isolation of the animals from the flock, J. Vet. Med. (A) (1986) 33:698 - 705.

[17] Buyse, J., Decuypere, E., Darras, V. M., Vleurick,L. M., Kuhn,E. R. and VeldhuisJ. D., Food deprivation and feeding of broiler chickens is associated with rapid and interdependent changes in the somatotrophic and thyrotrophic axes, Br.Poult. Sci. (2000) 41:107 - 116.

[18] Turkyilmaz, M.K., Nazligul, A.,Turkyilmaz, S. and Fidan, E., Effect of different feed withdrawals on performance and faecal contamination of carcass of broiler chickens, Int. J.Poult. Sci. (2006) 5:975 - 978.

[19] Lyon, C. E., Papa, C. M. and Wilson Jr, R. L., Effect of feed withdrawal on yields, muscle pH, and texture of broiler breast meat, Poult Sci. (1991) 70:1020 - 1025. 